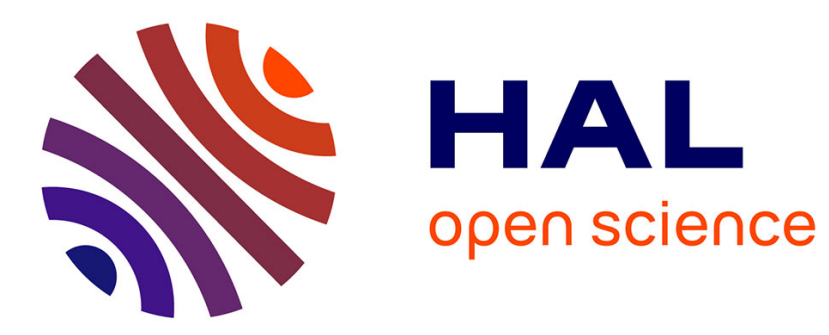

\title{
Statistical Lagrangian evaporation rate of droplets released in a homogeneous quasi-isotropic turbulence
}

\author{
L. Mees, N. Grosjean, Jean-Louis Marié, Corinne Fournier
}

\section{To cite this version:}

L. Mees, N. Grosjean, Jean-Louis Marié, Corinne Fournier. Statistical Lagrangian evaporation rate of droplets released in a homogeneous quasi-isotropic turbulence. Physical Review Fluids, 2020, 5 (11), 10.1103/PhysRevFluids.5.113602 . hal-02991507

\section{HAL Id: hal-02991507 \\ https://hal.science/hal-02991507}

Submitted on 6 Nov 2020

HAL is a multi-disciplinary open access archive for the deposit and dissemination of scientific research documents, whether they are published or not. The documents may come from teaching and research institutions in France or abroad, or from public or private research centers.
L'archive ouverte pluridisciplinaire HAL, est destinée au dépôt et à la diffusion de documents scientifiques de niveau recherche, publiés ou non, émanant des établissements d'enseignement et de recherche français ou étrangers, des laboratoires publics ou privés. 


\title{
Statistical Lagrangian evaporation rate of droplets released in a homogeneous quasi isotropic turbulence.
}

\author{
L. Méès, ${ }^{*}$ N. Grosjean, and J.L. Marié \\ Univ. Lyon, CNRS, Ecole Centrale de Lyon, INSA Lyon, Université Claude Bernard Lyon 1 \\ Laboratoire de Mécanique des Fluides et d'Acoustique, UMR 5509, F-69134, Ecully, France, \\ C. Fournier \\ Laboratoire Hubert Curien, UMR 5516, F-42023, Saint-Etienne, France \\ Univ Lyon, UJM-Saint-Etienne, CNRS, Institut d'Optique Graduate School
}

(Dated: November 6, 2020)

\begin{abstract}
Turbulence has long been suspected to increase the evaporation rate of droplets via the convective effects it generates. The experimental data reported in this paper provide evidence of this increase and statistically quantify these effects. Measurements have been performed following the same Lagrangian approach as in Chareyron et al. [1], Marié et al. [2,3]. Ether droplets have been released in a quasi isotropic homogeneous turbulence generated by synthetic jets and tracked using in-line digital holography. Their Schmidt number is typically of the order of 2 and their Reynolds number is moderate $(\leq 3)$. Their instantaneous positions and diameters have been measured by processing the holograms with an inverse problem approach (IPA) that has been implemented in Python language for a high performance computer. This has allowed to drastically reduce the processing time and to reconstruct a high number of trajectories for various turbulence conditions. The Lagrangian statistics computed from these trajectories, totaling 1.3 million samples, show that the relative mean motion and turbulence seen by the droplets on average increases their evaporation rate. Within the parameter range investigated, we find that this increase is not well predicted when estimating the convective effect in the Sherwood number with the norm of the instantaneous relative velocity seen by the droplets. In contrast, this increase is very well predicted when the Sherwood number is calculated using a Reynolds number based on the norm of the mean relative velocity plus its RMS fluctuation.
\end{abstract}

\section{INTRODUCTION}

Droplets evaporation occurs in numerous natural and industrial processes, covering a large variety of flow situations and scales (physics of clouds, atmospheric releases, spray dryers, agricultural spreading, motor engines...). In many situations the droplets vaporize in the turbulent gas flow carrying them and the question that naturally arises is whether or not the turbulence increases the evaporation rate [4]. In the absence of turbulence the evaporation rate of individual droplets in quasi-stationary conditions obeys the well-known " $d^{2}$ " law and in that case, the square of the droplet diameter $d$ decreases linearly with time [5]. The main parameters governing the evaporation rate are the temperature of the droplet and of the surrounding fluid, the mass fraction of vapor at the surface of the droplet and far away from it, and its velocity relative to the carrier mean gas flow (convective effect).

In a turbulent flow the droplets disperse spatially in time, which renders the heat and mass transfer with the gas flow much more complicated. By interacting with the turbulent eddies they cross, the droplets disperse following different trajectories [6, 7]. As a result, the surrounding instantaneous velocity fluctuations, temperatures and vapor mass fraction that they "sample" along their trajectories and that are likely to affect the evaporation rate, will differ from one droplet to another. The evaporation rate of each droplet is thus linked to its history from its injection [8]. This history is all the more complex because the size of the droplets decreases along their trajectory. This in turn has an effect on the way they disperse, hence on the evaporation. Depending on their Stokes number (the dimensionless time response of the droplet to the typical time scale of the turbulent

*1oic.mees@ec-lyon.fr flow velocity fluctuation), droplets may concentrate in preferential regions and form clusters [7], which may reduce the evaporation rate [9]. Experimental evidence of this effect was recently reported by Sahu et al. [10], who clearly showed that the droplet clustering in an acetone droplet spray leads to a reduction of the droplet evaporation rate. Their results indicate that the reduction is caused by a decrease of the inter-droplet distance and the resulting enrichment of vapor from neighboring droplets. Moreover they find that the dispersion of acetone droplets is different from that of same diameter water droplets with a slower evaporation rate.

Faced with this complexity, we chose to investigate the coupling between turbulence and evaporation in a simple and academic situation. It consists of releasing a diluted spray of droplets in a homogeneous isotropic flow field produced by synthetic jets $[1,11]$. The droplets number density being low, no clustering effects are expected and we can focus on the role of the instantaneous relative velocities seen by the droplets on their evaporation rate. The fluid selected after preliminary tests with freon [1], is diethyl ether, which evaporates fast in the atmospheric conditions of temperature and pressure where we work. We adopted a Lagrangian point a view like in turbulent dispersion studies $[6,12]$ that consists of tracking individual droplets and measuring the Lagrangian time evolution of their diameter along their trajectories. The tracking method that is used is in-line digital holography because it provides information on size and position using a rather simple optical device [13-16]. To obtain the accuracy of position and diameter required for the study of evaporation, the holograms recorded are reconstructed using an "inverse problem approach" (IPA) algorithm [17-20] that differs from the standard back propagation reconstruction methods. Two sets of experiments were performed up to now, one without turbulence (free-falling droplets [2]) and one with turbulence (synthetics jets working [3]). This second experiment has shown that the Lagrangian evaporation rate that is quasi constant in the free falling situation, may fluctuate and sometimes strongly increases in the presence of turbulence. Unfortunately 
this was shown on a very limited number of trajectories so that no statistical quantitative conclusion could be drawn.

The objective of this paper is therefore to confirm these results by measuring the statistical Lagrangian evaporation rate for a large number of trajectories and for a wider range of turbulent flow conditions. This requires to handle two main difficulties: increasing the number of droplets imaged in the turbulence domain and decreasing drastically the processing time of the holograms. The first difficulty was solved by reducing the magnification of our holographic optical setup and optimizing the location of the droplets release. The second one was overcome by using parallel computing on a high performance computer to process the holograms. The features of the experiments (turbulence flow, droplet injection, holographic optical set-up and inverse-method used to process the holograms) have been detailed in our previous papers [1-3]. Thus, they will only be briefly presented, while we focus on improvements and new results. Section II provides some review of the experimental set-up and procedures. It is followed by a section that describes how the hologram processing time was strongly reduced (section III). The Lagrangian statistics are then reported and commented (section IV). Within the range of parameter explored (Schmidt number $\approx 2$, droplets Reynolds number $\leq 3$ ), they clearly show that the turbulence velocity seen by the droplets increase the evaporation rate.A formulation accounting for this effect in the frame of the film theory (Bird et al. [21], Abramzon and Sirignano [22]) is proposed and commented. The paper ends with a summary of the main findings.

\section{EXPERIMENTAL SET-UP AND PROCEDURES}

A sketch of the experimental arrangement: woofers, holographic optical set-up axes and droplets injector, is displayed in figure 1 .

\section{A. Turbulence characteristics}

The turbulence is created by the meeting of three pairs of opposing synthetic jets produced by woofers, as described in Goepfert et al. [11]. This system generates a homogeneous, nearly isotropic turbulence whose properties are close to those of the 'box of turbulence' imagined by Hwang and Eaton [23]. A similar system was operated some time ago in a four pair configuration by Lian et al. [24] with comparable turbulence characteristics and more recently in a quasi identical three pair configuration by Patel and Sahu [25]. The turbulence domain produced is a box of about $50 \mathrm{~mm}^{3}$, located $340 \mathrm{~mm}$ from each woofer and centered on the stagnation point of the opposing jets. To obtain a good zero mean velocity within the box, this stagnation point must be stable, which requires balancing each pair of opposing jets at the center of the box. This is done by finely tuning the amplitude of the $50 \mathrm{~Hz}$ woofers sinusoidal driving signals using an iterative procedure. The balance is then kept in time by adjusting these amplitudes continuously so that the pressure signals of the sensors installed on each woofer exit remain constant within $\pm 0.2 \%$ standard deviation [3]. The level of turbulence within the box, and its characteristics scales are fixed by the amplitude of the sinusoidal driving signal. They are measured using two-velocity component laser Doppler velocimetry (LDV) and particle imaging velocimetry (PIV) following the procedure described in Chareyron et al. [1]. The experiments reported in Marié et al. [3] have been performed in turbulence with a mean flow velocity, RMS velocity fluctuations and an integral length scale of the order of $0.03 \mathrm{~ms}^{-1}, 0.8 \mathrm{~ms}^{-1}$ and $49 \mathrm{~mm}$ respectively. Its characteristics are summarized in table I. Here, the

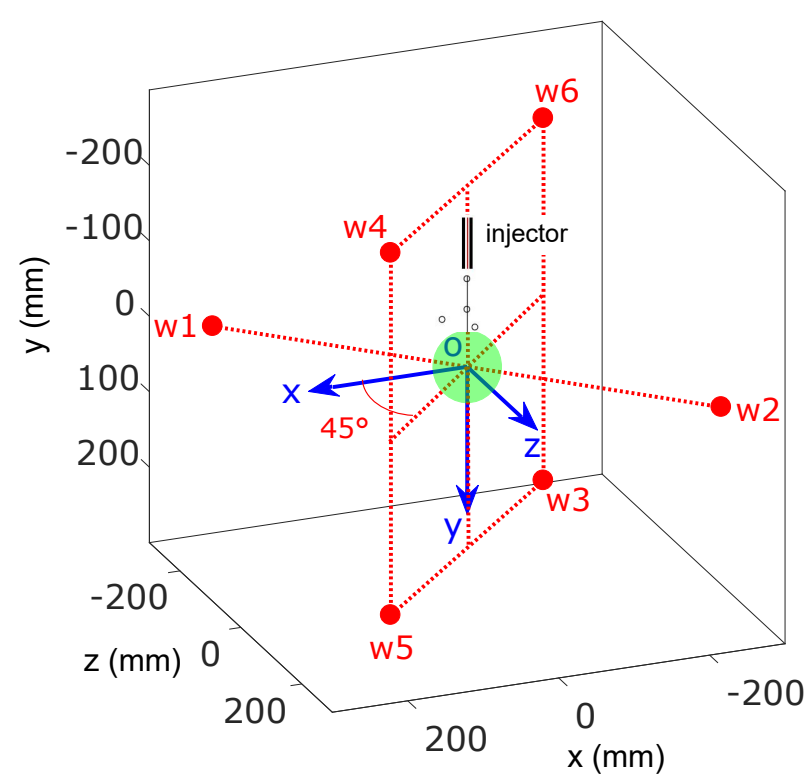

FIG. 1. Sketch of the woofers (red spheres) and of the holographic optical set-up axes (blue axes); distance between woofers of each pair: $680 \mathrm{~mm}$; the angle between each pair is $90^{\circ}$; $\mathrm{z}$ is the optical axis of the in-line holographic set-up; $y-z$ are the 2 axes of the twovelocity component LDV system used to measure the velocity in the turbulence box; the green sphere represents the $50 \mathrm{~mm}^{3}$ turbulence box; the injector is positioned on the y axis.

evaporation of ether droplets have been studied for the same conditions (two runs noted 1 and 2 for this reference situation), plus four other RMS turbulent velocities: $0.2 \mathrm{~ms}^{-1}, 0.4 \mathrm{~ms}^{-1}, 0.6 \mathrm{~ms}^{-1}$ and $1.0 \mathrm{~ms}^{-1}$ (table II), in order to quantify the influence of these turbulent velocities on the evaporation rate. The corresponding Reynolds number based on the Taylor micro scale $R e_{\lambda}$ were not systematically determined. However, referring to the results of Lian et al. [24] obtained with a similar system, we may expect $R e_{\lambda}$ to vary between 50 and 250 .

\section{B. Droplets injection}

The droplets are generated by a piezoelectric MJ-AT-01 MicroFab Technologies jetting device, equipped with a $60 \mu \mathrm{m}$ orifice diameter glass capillary. The device was used in the "continuous jet-mode" in Marié et al. [3], whereas it is operated here in the "drop-on-demand mode" like in Chareyron et al. [1], Marié et al. [2]. In this mode, the ether droplets are delivered by the contraction of the glass capillary at the selected piezoelectric frequency and their initial diameter is more stable and of the order of the orifice diameter [26, 27]. The droplet exit velocity is also lower, of the order of $1 \mathrm{~ms}^{-1}$, so that the injector can be positioned closer to the box of turbulence than with the "continuous jet-mode". This allows the number of droplets entering the turbulence domain to be better controlled and thus, the number of droplets imaged inside it increases. Droplets are released above the turbulence box at a point on the vertical $y$-axis (figure 1), but as illustrated in figure 2(a), many of them are expelled outside by the turbulence. Therefore, the injection distance from the centre of the turbulence box (about $25 \mathrm{~mm}$ ) was adjusted to obtain a significant 
TABLE I. Turbulence characteristics in Marié et al. [3].

\begin{tabular}{|c|c|c|c|c|c|c|c|}
\hline $\begin{array}{l}\text { twice the } \\
\text { kinetic energy }\end{array}$ & $\begin{array}{l}\text { dissipation } \\
\text { rate }\end{array}$ & $\begin{array}{l}\text { Taylor } \\
\text { micro-scale }\end{array}$ & $\begin{array}{l}\text { Reynolds } \\
\text { number }\end{array}$ & $\begin{array}{l}\text { turnover } \\
\text { time }\end{array}$ & $\begin{array}{l}\text { integral } \\
\text { length scale }\end{array}$ & $\begin{array}{l}\text { Kolmog } \\
\text { length scale }\end{array}$ & $\begin{array}{l}\text { gorov } \\
\text { time scale }\end{array}$ \\
\hline$q^{2}\left(m^{2} s^{-2}\right)$ & $\varepsilon\left(m^{2} s^{-3}\right)$ & $\lambda(\mathrm{mm})$ & $\operatorname{Re}_{\lambda}$ & $T_{E}(m s)$ & $\Lambda(m m)$ & $\eta_{k}(m m)$ & $\tau_{k}(m s)$ \\
\hline 2.10 & 7.6 & 5.1 & 229 & 138 & 49 & 0.15 & 1.56 \\
\hline
\end{tabular}

TABLE II. Flow conditions.

The time average velocities and fluctuations are those measured by LDV at the centre of the domain.

\begin{tabular}{|c|c|c|c|c|c|c|c|c|}
\hline run & $\begin{array}{l}\text { ambient } \\
\text { temperature }\end{array}$ & $\begin{array}{l}\text { ambient } \\
\text { relative } \\
\text { humidity }\end{array}$ & $\begin{array}{l}\text { average } \\
\text { initial droplet } \\
\text { diameter }\end{array}$ & $\begin{array}{l}\text { average } \\
\text { y-velocity }\end{array}$ & $\begin{array}{l}\text { average } \\
\text { z-velocity }\end{array}$ & $\begin{array}{l}\text { RMS } \\
\text { y-velocity } \\
\text { fluctuation }\end{array}$ & $\begin{array}{l}\text { RMS } \\
\text { z-velocity } \\
\text { fluctuation }\end{array}$ & $\begin{array}{l}\text { twice the } \\
\text { kinetic energy }\end{array}$ \\
\hline & $T^{\infty}\left({ }^{\circ} C\right)$ & $R_{h}^{\infty}(\%)$ & $\overline{d_{I}}(\mu m)$ & $\overline{U_{y}}\left(m s^{-1}\right)$ & $\overline{U_{z}}\left(m s^{-1}\right)$ & $\sqrt{u_{y}^{2}}\left(m s^{-1}\right)$ & $\sqrt{u_{z}^{2}}\left(m s^{-1}\right)$ & $q^{2}\left(m^{2} s^{-2}\right)$ \\
\hline Free fall & 25.0 & 36.1 & 41.7 & - & - & - & - & - \\
\hline RMS 0.2 & 28.0 & 32.9 & 71.1 & 0.04 & 0.01 & 0.21 & 0.21 & 0.13 \\
\hline RMS 0.4 & 28.1 & 33.2 & 66.6 & 0.13 & -0.02 & 0.39 & 0.38 & 0.44 \\
\hline RMS 0.6 & 27.6 & 33.8 & 61.8 & 0.07 & -0.10 & 0.58 & 0.60 & 1.05 \\
\hline RMS $0.8^{1}$ & 27.0 & 35.0 & 59.5 & -0.00 & 0.04 & 0.75 & 0.83 & 1.88 \\
\hline RMS $0.8^{2}$ & 27.9 & 31.5 & 70.4 & 0.08 & -0.03 & 0.80 & 0.80 & 1.92 \\
\hline RMS 1.0 & 27.6 & 34.0 & 65.8 & -0.04 & 0.04 & 1.02 & 1.01 & 3.09 \\
\hline
\end{tabular}

fraction of droplets entering the box. As noted in Chareyron et al. [1], turbulence slightly influences the size distribution at the exit of the orifice. This size that well peaked around $60 \mu \mathrm{m}$ without turbulence ranges between $50-70 \mu \mathrm{m}$ when the woofers are working. The number of droplets recorded within the turbulence on average per hologram never exceeds 5-10, meaning that droplets are sufficiently sparse to consider that they evaporate without interaction. The fluid injected is diethyl ether manufactured by Sigma-Aldrich. It contains less than $0.1 \%$ residual water. Droplets are released at ambient temperature and pressure. Air temperature and relative humidity in the room are controlled with an air-conditioner and a dehumidifier, and measured for each run. Their values are reported in table II. Despite our efforts to keep them constant, room temperature and relative humidity show small variations from one run to another. The effect of these variations on evaporation will be commented in the last section. Also, table II provides the average initial diameter $\bar{d}_{I}$ of droplets that are tracked in a run.

The droplets being smaller than in Marié et al. [3] due to the injection mode adopted, their time response $\tau_{d}=\rho_{e} d^{2} / 18 \mu_{g}^{r}$ to a sudden change in the surrounding velocity is between 7-14 ms, against 10$30 \mathrm{~ms}$ previously. As a result, their turbulent Stokes number $\tau_{d} / \tau_{\eta}$ in the same turbulence condition (run RMS 0.8) is lower: between 4-9, against 7-20. The characteristic evaporation time $\tau_{e v}$ of the droplet defined as $\bar{d}_{I}^{2} / K_{s}$, with $K_{s}=-\dot{d}^{2}$, the surface evaporation rate is typically $120 \mathrm{~ms}$. This time is of the same order of magnitude as the eddy turnover time $T_{E}=138 \mathrm{~ms}$ for the reference turbulent flow conditions (RMS 08). The resulting Damkhöler number $D a=T_{E} / \tau_{e v}$ is of the order of one, suggesting that we are in a situation where the turbulence can influence droplet evaporation [4].

\section{Digital Holographic set-up}

Figure 3 provides a schematic of the in-line holographic optical set-up. The turbulence domain is illuminated by a laser beam (wavelength $\lambda=532 \mathrm{~nm}$ ) produced by a Nd:YVO4 solid laser Millenia IIs (a)

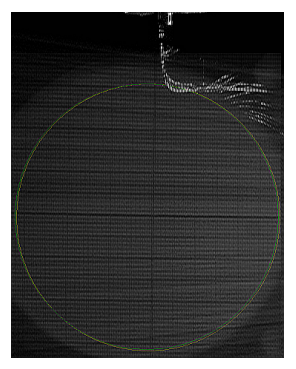

(b)
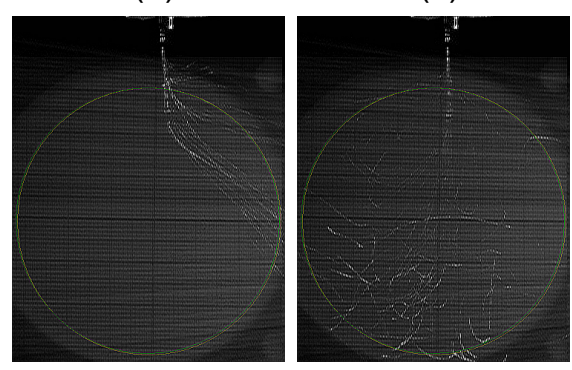

FIG. 2. A sequence illustrating the droplets jet behavior at the top of the turbulence box; the large circle materializes the edge of the turbulence box; the sequence was recorded with a $30 \mathrm{fps}$ camera. (a): no entering, (b) partial entering, (c) full entering and dispersion.

Spectra Physics. To improve the uniformity of the lighting, the beam at the laser exit is focused by a converging lens onto a pinhole placed at its focal lens $f_{1}=25.4 \mathrm{~mm}$. Unlike in Marié et al. [3] where a divergent set-up was used, the beam is then collimated towards the domain of measurement by a second lens placed at its focal lens $f_{2}=400 \mathrm{~mm}$ from the pinhole. This new set-up allowed to image a larger part of the turbulence domain, which is essential to record longer trajectories and to increase the Lagrangian statistics. The imaged domain is a rectangular cuboid $x \times y \times z$ of about $16 \times 25.6 \times 50$ $\mathrm{mm}^{3}$, significantly larger than the previous one $\left(10 \times 16 \times 50 \mathrm{~mm}^{3}\right.$ in Marié et al. [3]). As shown in Chareyron et al. [1], the use of IPA to process the holograms enables to detect droplets outside of the field of view up to an effective cross size as large as $42 \mathrm{~mm} \times 40 \mathrm{~mm}$. This potential has not been used here because the thermal/vapour films created by evaporation around the droplets generate hologram modifications [28, 29], which render the outfield detection more difficult and less accurate. The holograms of droplets are recorded at $6.2 \mathrm{kHz}$ framerate on the $800 \times 1280$ pixels CMOS sensor of a Phantom V611 camera, with pixels size of $20 \mu \mathrm{m}$ and a fill-factor of 0.56 . 


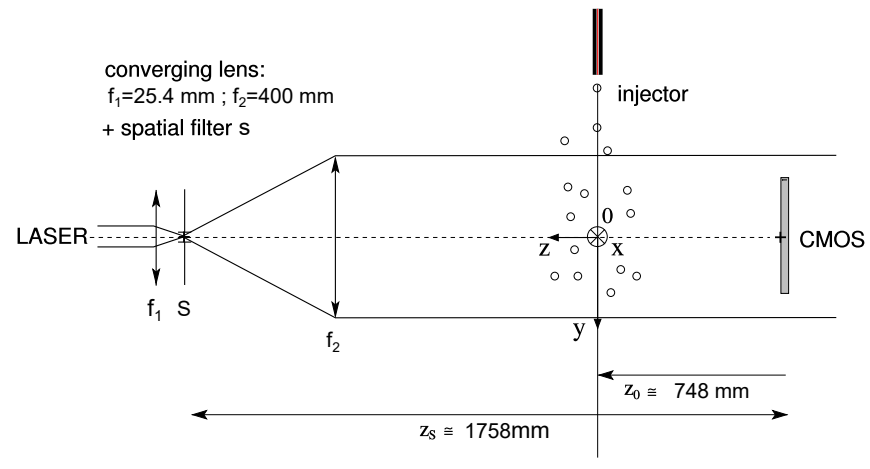

FIG. 3. Holographic optical set-up. The coordinate axis are the same as in figure 1

The sensor is positioned at a distance $z_{0}=748 \mathrm{~mm}$ from the center of the turbulence domain $(x=y=z=0$, figure 1$)$. The magnification factor $m$ of the collimated optical set-up is nearly 1 (1.0013). It has been deduced from calibration by using a linear scale glass reticle (Edmunds Optics, $\sharp 62-252$ ), following the same procedure as described in Marié et al. [2].

\section{HOLOGRAM PROCESSING}

\section{A. Principle of the 3D droplet reconstruction with the IPA}

The inverse problem approach (IPA) was preferred to a standard back light propagation procedure to extract the coordinates and the diameter of the droplets. The reason is that it provides high accuracy on droplet position and size required to track them and to measure their evaporation rate at relatively large distances in a large measurement volume. The IPA for hologram reconstruction is described in Soulez et al. [17, 18] and successfully applied to droplet tracking in Chareyron et al. [1], Marié et al. [2, 3], Seifi et al. [28]. It consists of minimizing the root mean square difference between each recorded hologram and a model hologram. The model that describes a spherical droplet hologram at large distance with enough accuracy is the scalar diffraction model of an opaque disk with Fraunhofer approximation. It only depends on the diameter $d_{k}$ and location $x_{k}, y_{k}, z_{k}$ of the droplets [30]. Minimizing the difference is thus equivalent to find iteratively within a $4 \mathrm{D}$ search space, the set of parameters $x_{k}, y_{k}, z_{k}, d_{k}$ producing the model hologram that best matches each individual droplet hologram in the image. In contrast to the classical back light propagation reconstruction process, the model hologram intrinsically takes into account truncation (finite size of the sensor) and low spatial resolution of the sensor (integration over a finite sized pixel), which are significant sources of error and lower accuracy of the measurements [19]. The accuracy of IPA for tracking droplets in the turbulence has been discussed in Chareyron et al. [1].

The vapor generated by the droplet evaporation induces refractive index gradients near the droplet surface[29] that are not taken into account in the diffraction model. To avoid any measurement inaccuracy associated with these gradients, the central part of each individual droplet hologram is masked as described in Seifi et al. [28], so that this part of the image is ignored from the fitting procedure. Here, the circular mask radius has been set to 75 pixels, in order to remove the three first fringes which have been proven to be the most sensitive to the presence of vapor[29]. Figure 4 provides an illustration of the hologram processing. An example of hologram with three evaporating droplets diffraction patterns after a background correction is given in figure 4(a). Its processing with the IPA and the mask method yields the best fit synthetic hologram displayed in figure 4(b). The residual obtained by subtracting the best fit hologram from the experimental one in figure 4(c) materializes the modification of the first fringes by the vapor that is not included in the diffraction model. Signal integration over the effective pixel area (the fill factor of the sensors' pixels is 0.56 ) is taken into account in the model holograms. The initial guess on parameters $x, y, z, d$ required as input for the IPA process is obtained by a pre-processing based on the correlation product between the experimental hologram and the model with sampled values of $z$ and $d$ (typically $31 \times 6$ ), $z$ ranging from $0.3 \mathrm{~m}$ to $0.8 \mathrm{~m}$ and $d$ ranging from $5 \mu \mathrm{m}$ to $200 \mu \mathrm{m}$. The maximum of correlation provides the initial guess for the droplet parameters and the IPA process provides the accurate final estimations. At this step, the model diffraction pattern computed from the parameters final estimations is subtracted from the experimental hologram to allow the next droplet detection and measurement following an optimal iterative process (greedy algorithm).

\section{B. Description of the fast 3D reconstruction and droplets tracking}

To track the droplets, sequences of about $0.9 \mathrm{~s}$ (5477 frames) were recorded on the high speed sensor and processed. For each hologram of a given sequence, diffracting objects were detected and the 4 parameters $(x, y, z, d)$ characterizing them were measured. The concentration of droplets being low, their tracking from frame to frame was achieved by using a simple 4D nearest-neighbor algorithm. Several trajectories are generally reconstructed from each hologram sequence but some of them are too short to be analysed and to extract statistically relevant measurements. This is the reason why only the trajectories longer than 200 frames $(\approx 32 \mathrm{~ms})$ were kept. Also, this selection makes it possible to detect and delete the trajectories of solid particles inside the turbulence domain (less than 4\%) and residual water drops, whose sizes remain almost constant over time. These drops are constituted by the water of the humid air that condensed on the cold ether droplets surface (temperature between -10 and $-15{ }^{\circ} \mathrm{C}$ ) during their fast vaporization and that remains after all the ether vaporized [2].

The main limitation of holography is the processing time, especially when IPA is adopted. Here, the CPU time required to process a single hologram containing 10 droplets is about 10 minutes. Each hologram sequence of 0.9 seconds (5477 frames) then requires a thousand of hours on a single CPU unit. The 554 sequences recorded for our statistics thus represent more than 3 Millions holograms that would required 57 years to be processed on a single CPU. This limitation has been pushed back by implementing the processing code in Python language for a High Performance Computing system (HPC), reducing the effective computing time to about 1 day per sequence when a single node with 32 cores is used. Another source of time consumption are the empty frames that may occur in hologram sequences. In free falling conditions, all frames contains droplets holograms [2]. This is no longer the case when a low number density of droplets disperse in the turbulence. In Marié et al. [3] the parts of the sequences containing droplets were manually selected to focus only on the longest and most interesting trajectories. Here, to fasten the computation while preserving the process from any operator dependency, a pre-processing based on the reduction of hologram resolution has been implemented. This technique is inspired from the multi-resolution approach proposed by Seifi et al. [31] to accelerate the hologram processing with IPA. The spatial resolution was 


\section{LAGRANGIAN STATISTICS}
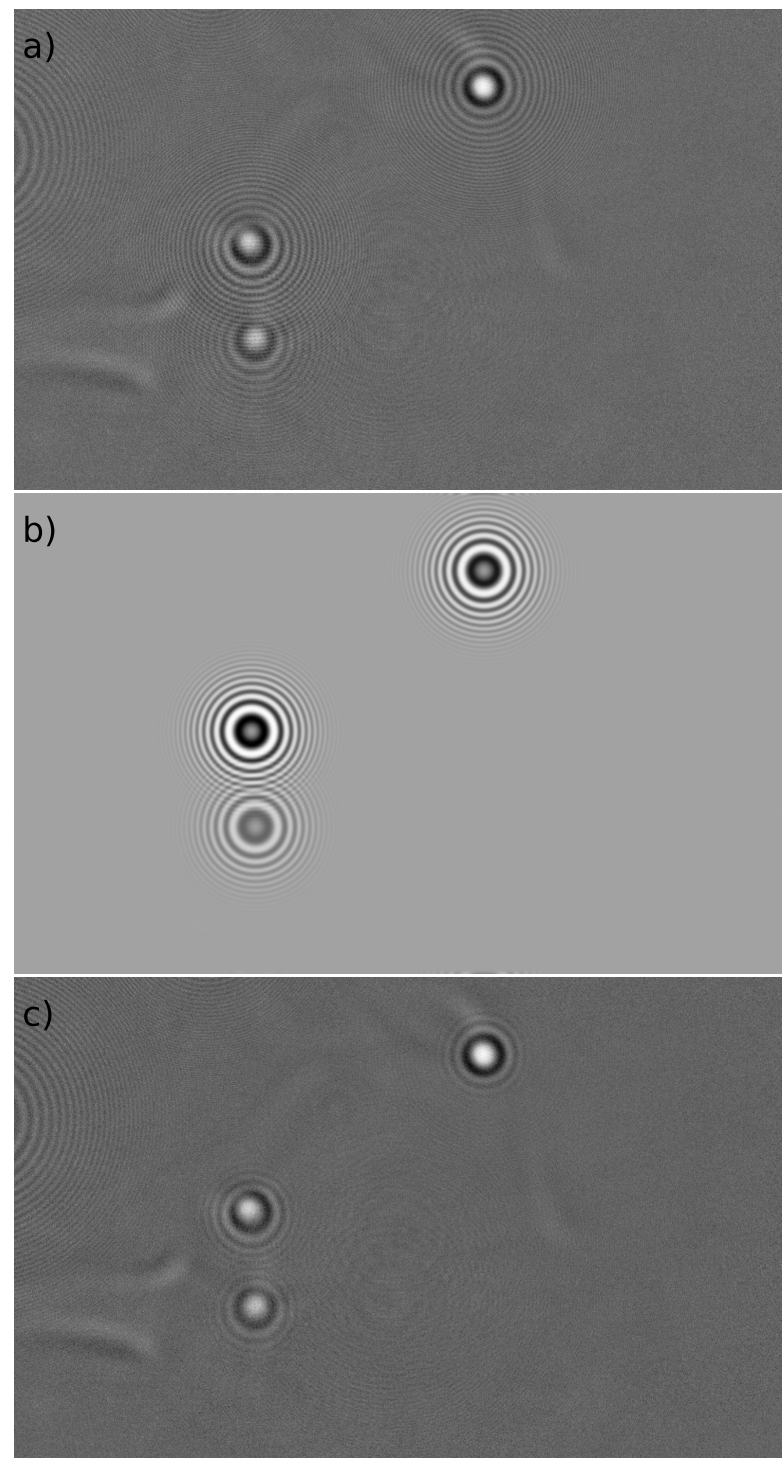

FIG. 4. In-line hologram of three evaporating droplets. The recorded hologram (a) after background correction is compared to the synthetic hologram that best matches it (b) and to the residual (c) obtained by subtracting the best fit hologram from the recorded hologram.

reduced by a factor 4 along $x$ and $y$ by using a binning process on the holograms and the temporal resolution was reduced by the same factor, i.e. selecting one in four images. All the droplets trajectories are detected and roughly characterised from the low resolution holograms in about one hour, allowing the selection of the useful frames to be processed with full resolution. The total gain obviously depends on the percentage of images kept for full processing. For the sequences processed for this paper, this percentage varies from $44 \%$ to $78 \%$, leading to an average gain in time of $43 \%$. Finally, the total CPU time used for this study was about 350000 hours, spread out onto different nodes of 32 or 16 cores.
The total number of trajectories that have been reconstructed to build our Lagrangian statistics is 4337, representing 1347960 time samples. Each trajectory provides the time evolution of the 3 spatial coordinates $x(t), y(t), z(t)$ and the diameter $d(t)$ of the droplet. The velocities and accelerations of the droplet along the track have been computed by differentiating the droplet coordinates, once and twice, respectively, after applying a Gaussian filter in frequency domain of the form

$$
G(v)=e^{-v^{2} / 2 v_{c}^{2}}
$$

to reduce noise effects. This kind of filtering is currently used in tracking techniques [32] to reduce the noise that results when differentiating positions to obtain velocities and accelerations. With in line holography, it also allows to decrease the uncertainty on the depth position to a value approaching that of the lateral directions, as explained in Chareyron et al. [1]. The cutting frequency of the Gaussian filter that has been selected is $v_{c}=75 \mathrm{~Hz}$. It is of the same order of magnitude as the inverse of the time response of the droplets investigated in this study. It turns out a good compromise to efficiently filters the noise while respecting the droplets dynamics. The instantaneous evaporation rate has been computed by differentiating the diameter square $d^{2}(t)$, after applying the same Gaussian filter.

The Lagrangian instantaneous relative velocity along the droplet trajectory involved in the convective effect is defined as $\mathbf{U}_{\mathbf{r}}=\mathbf{U}_{\mathbf{d}}-\mathbf{U}$, with $\mathbf{U}_{\mathbf{d}}$ the droplet velocity and $\mathbf{U}$ that of the surrounding gas velocity. This relative velocity cannot be directly measured with the holographic set-up. However, $\mathbf{U}_{\mathbf{r}}$ can be reasonably well inferred from droplet position and diameter measured by digital holography, via the equation of motion of the droplet [1]. It consists in finding at each time step the relative velocity that is consistent with the positions and diameters measured by holography, as predicted by this equation. The relevancy of the Lagrangian relative velocity thus calculated is based on the fact that the components of this velocity are aligned with the vapor wake visible behind each droplet holograms $[1,3]$.

The Probability Density Functions (PDFs), the average and the standard deviation of the quantities relevant for the study, namely the droplet velocity and acceleration components, the relative velocity components and the instantaneous evaporation rate, have been calculated for each of the flow conditions investigated (table II). Each quantity $\mathrm{Q}$ is decomposed into the sum of a mean value $\bar{Q}$ and a fluctuation $q$, where $\bar{Q}$ designates the statistical average over all the samples of a given flow condition (number of trajectories $\times$ number of points per trajectory). The RMS value of the fluctuation is noted $q^{\prime}=\sqrt{\overline{q^{2}}}$

\section{A. Results}

Figure 5(a) shows that the PDFs of the normalized droplet velocity components obtained for a high $R e_{\lambda}$ conditions (RMS $0.8^{1}$ ) are almost Gaussian, with standard deviations which are relatively close to each other (table III). However, the mean velocity in $y$ direction is not as small as in other directions, as expected in a quasi isotropic homogeneous turbulence with zero mean flow. The reason is that droplets are released at the top of the turbulence box and that they can enter the box when the turbulent flow facing them is not too strong or oriented downward (see figure $2 \mathrm{c}$ ). This non-isotropic injection condition also slightly modifies the shape of the $y$ velocity component PDF and explains the small asymmetry in figure 5(a). The PDFs of the acceleration components are presented in figure 5(b). As can 
be seen, the three components normalized by their RMS values superpose rather well. Their shape resembles the ones reported for fluid particles at this $R e_{\lambda}$ by Mordant et al. [32], Voth et al. [33] and agree well with the statistical distribution found by these authors for the normalized acceleration components:

$$
P\left(x_{i}\right)=\frac{e^{3 s^{2} / 2}}{4 \sqrt{3}}\left[1-\operatorname{erf}\left(\frac{\ln \left(\left|x_{i}\right| / \sqrt{3}\right)+2 s^{2}}{\sqrt{2} s}\right)\right]
$$

This distribution supposes that components are isotropic and that the magnitude is log-normal. $s$ is a parameter related to the global shape of the PDF [34]. A close agreement with our data has been obtained taking $s=0.62$. This value turns to be the best-fit value found by Qureshi et al. [35] for inertial particles over a wide range of sizes and densities and with Stokes number between 1-40. This result is even more interesting since in our case the Stokes number of evaporating droplets varies in time. Compared to the measurements of these authors, the tails of the statistics distribution do not extend very far, the mean reason being that the scarce strong events whose probability is smaller than $10^{-3}$ can hardly be measured in our experimental conditions [1]. The average and standard values of the droplets velocity components for the other RMS turbulence values are reported in table III.

The PDFs of the normalized relative velocity components, inferred from the equation of motion for the run RMS $0.8^{1}$ are plotted in Figure 6. Again, the three components superposes rather well, with a better symmetry in $x$ and $z$ directions, as expected due to our injection conditions. As noted in table III, $\overline{U_{r x}}$ and $\overline{U_{r z}}$ are close to zero while $\overline{U_{r y}}$ is slightly negative. These trends are observed for the other RMS turbulent velocities and reflect the non isotropic character of the injection. It confirms that droplets on average enter the domain when the turbulent velocity is oriented downward and is higher than the droplet velocity. The standard deviations in the three directions are relatively close to each other and on average smaller than those of the droplet velocity components. The shape of these PDFs in the $x$ and $z$ directions turns out to be similar to the one observed for the droplets acceleration components.

Finally, figure 7 shows the PDFs of the evaporation rate $K_{s}$ for the various RMS turbulent velocities. Under their non-normalized form (figure 7a), we note that that both the average and the RMS fluctuation of the evaporation rate tend to increase with the RMS turbulent velocity, but that this increase is not monotonic. This is confirmed in table IV where the corresponding values are reported. As explained in the next section, the reason of this behavior is that $K_{s}$ is influenced both by the mean and the fluctuating relative velocity seen by the droplets and that these quantities do not always increase monotonically with the RMS turbulent velocity. Under their normalized form (figure 7b) all these PDFs collapse fairly well onto a single distribution. Interestingly, the shape of this distribution is the same as the one of the PDFs of the droplet normalized acceleration components.

\section{B. Statistical turbulent convective effect}

Without turbulence (Marié et al. [2]), we have shown that the Lagrangian evaporation rate of ether droplets is quasi constant like in a classical " $\mathrm{d}^{2}$ " law. This was validated by a simple model, in which the transfers between the droplets and the surrounding gas are expressed in the frame of the film theory (Bird et al. [21], Abramzon and Sirignano [22]). All processes in this model are supposed to be quasi-steady, and the thermal and mass concentration boundary layers developing around the droplets are modeled by two spherical films, whose thicknesses account for the Stefan flux and the convec-
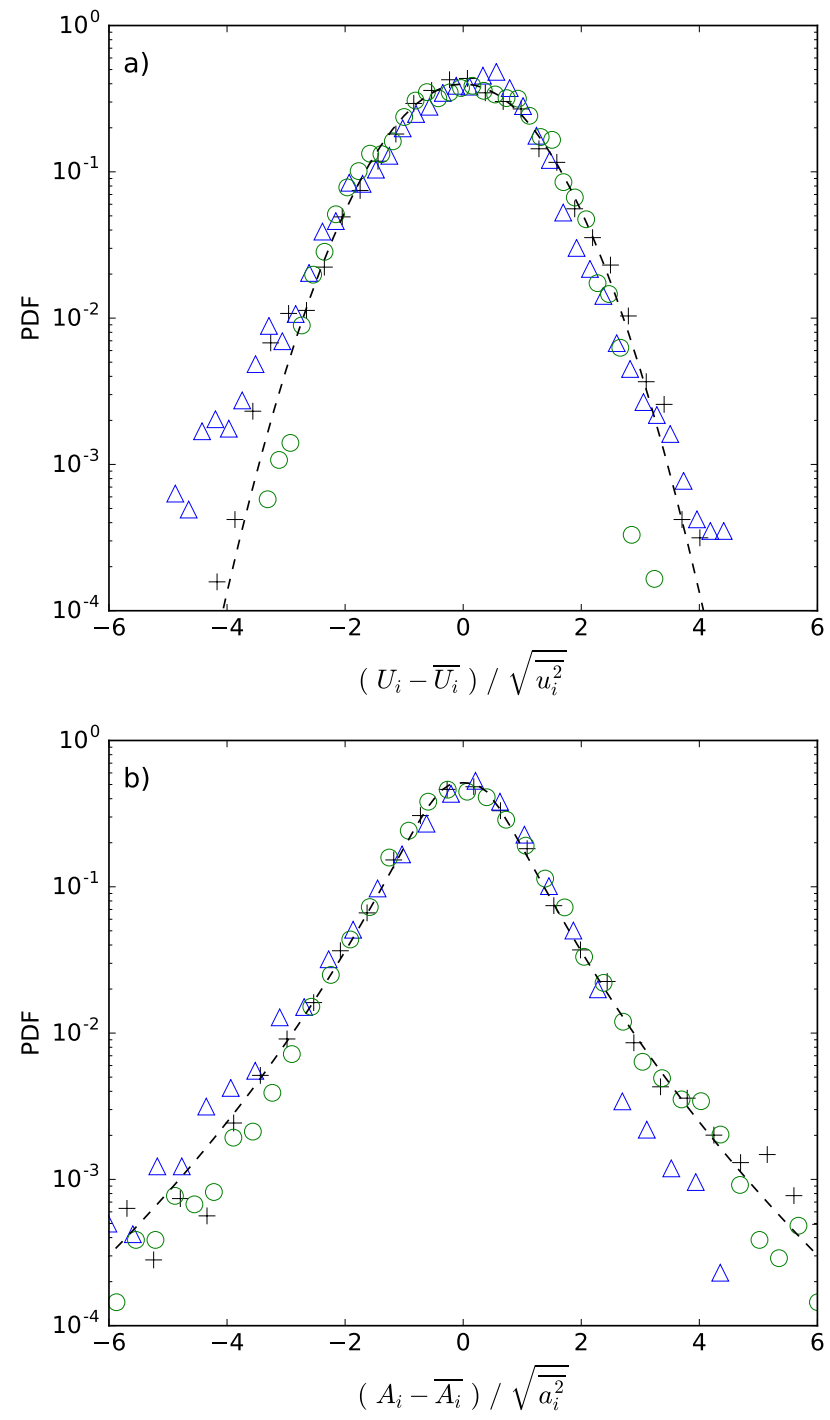

FIG. 5. Probability density functions of (a) the normalized droplets velocity components, $+: x$ direction, $\triangle: y$ direction, $\circ: z$ direction, dashed line: normal distribution; (b) the normalized acceleration components, same symbols and colors, dashed line: equation (1) with $\mathrm{s}=0.62$.

tive effects of the external flow. The corresponding Lagrangian mass evaporation rate is given by

$$
\dot{m}_{e}=\frac{d m_{e}}{d t}=\pi \rho_{g} d D_{e} \log \left(1+B_{M}\right) S h_{F}
$$

where $D_{e}$ is the mass diffusivity of the Diethyl Ether vapor in the air, $\rho_{g}$ the surrounding gas film density, $B_{M}$ and $S h_{F}$ the Spalding and the convective Sherwood numbers, respectively. The Spalding number, expressed as $\left(Y_{e}^{S}-Y_{e}^{\infty}\right) /\left(1-Y_{e}^{S}\right)$, is calculated by taking the vapor mass fraction at the surface droplet $Y_{e}^{s}$ under the saturation conditions corresponding to droplet temperature $T^{s}$. The vapor fraction far away from the droplet $Y_{e}^{\infty}$ is assumed to be constant, equal to zero, which is justified by the fact that droplets are very diluted. The convective 
TABLE III. Droplets and relative velocity components, average values and standard deviations.

\begin{tabular}{lllllllllllll}
\hline run & $\overline{U_{x}}$ & $\overline{U_{y}}$ & $\overline{U_{z}}$ & $\sqrt{\overline{u_{x}^{2}}}$ & $\sqrt{\overline{u_{y}^{2}}}$ & $\sqrt{\overline{u_{z}^{2}}}$ & $\overline{U_{r x}}$ & $\overline{U_{r y}}$ & $\overline{U_{r z}}$ & $\sqrt{\overline{\overline{u_{r x}^{2}}}}$ & $\sqrt{\overline{\overline{u_{r y}^{2}}}}$ & $\sqrt{\overline{u_{r z}^{2}}}$ \\
\hline Free fall & -0.012 & 0.104 & 0.014 & 0.023 & 0.058 & 0.028 & 0.004 & 0.004 & -0.004 & 0.014 & 0.028 & 0.04 \\
RMS = 0.2 & 0.024 & 0.238 & 0.028 & 0.104 & 0.179 & 0.173 & 0.002 & -0.09 & 0.011 & 0.029 & 0.081 & 0.118 \\
RMS = 0.4 & 0.018 & 0.306 & 0.16 & 0.163 & 0.303 & 0.237 & -0.015 & -0.12 & 0.015 & 0.053 & 0.106 & 0.161 \\
RMS = 0.6 & -0.035 & 0.388 & 0.027 & 0.228 & 0.307 & 0.331 & -0.016 & -0.087 & 0.009 & 0.08 & 0.094 & 0.157 \\
RMS = 0.8 & -0.021 & 0.402 & -0.025 & 0.263 & 0.354 & 0.415 & -0.005 & -0.093 & 0.017 & 0.107 & 0.128 & 0.163 \\
RMS = $0.8^{2}$ & -0.016 & 0.363 & -0.053 & 0.266 & 0.387 & 0.429 & -0.008 & -0.156 & 0.018 & 0.144 & 0.198 & 0.293 \\
RMS = 1.0 & -0.056 & 0.339 & -0.082 & 0.278 & 0.455 & 0.459 & -0.015 & -0.145 & -0.01 & 0.145 & 0.203 & 0.225 \\
\hline
\end{tabular}

TABLE IV. Mean Lagrangian statistics.

\begin{tabular}{|c|c|c|c|c|c|c|c|c|}
\hline run & $\begin{array}{l}\text { ambient } \\
\text { temperature }\end{array}$ & $\begin{array}{l}\text { ambient } \\
\text { relative } \\
\text { humidity }\end{array}$ & $\begin{array}{l}\text { average } \\
\text { initial droplet } \\
\text { diameter }\end{array}$ & $\begin{array}{l}\text { average } \\
\text { relative } \\
\text { velocity }\end{array}$ & $\begin{array}{l}\text { RMS } \\
\text { relative } \\
\text { fluctuation }\end{array}$ & $\begin{array}{l}\text { average } \\
\text { evaporation } \\
\text { rate }\end{array}$ & $\begin{array}{l}\text { RMS } \\
\text { evaporation } \\
\text { fluctuation }\end{array}$ & $\begin{array}{l}\text { average } \\
\text { Reynolds } \\
\text { number }\end{array}$ \\
\hline & $T^{\infty}\left({ }^{\circ} \mathrm{C}\right)$ & $R_{h}^{\infty}(\%)$ & $\overline{d_{I}}(\mu m)$ & $\overline{U_{r}}\left(m s^{-1}\right)$ & $\sqrt{\overline{u_{r}^{2}}}\left(m s^{-1}\right)$ & $\overline{K_{s}}\left(m^{2} s^{-1}\right)$ & $\sqrt{k_{s}^{2}}\left(m^{2} s^{-1}\right)$ & $\overline{R e_{d}\left(U_{r}\right)}$ \\
\hline Free fall & 25.0 & 36.1 & 41.8 & 0.011 & 0,010 & $3.13 \mathrm{e}-8$ & $5.11 \mathrm{e}-9$ & 0.03 \\
\hline RMS 0.2 & 28.0 & 32.9 & 71.1 & 0.124 & 0.119 & $3.74 \mathrm{e}-8$ & $1.34 \mathrm{e}-8$ & 0.64 \\
\hline RMS 0.4 & 28.1 & 33.2 & 66.6 & 0.178 & 0.152 & $3.98 \mathrm{e}-8$ & $1.55 \mathrm{e}-8$ & 0.86 \\
\hline RMS 0.6 & 27.6 & 33.8 & 61.8 & 0.178 & 0.126 & $3.80 \mathrm{e}-8$ & $1.49 \mathrm{e}-8$ & 0.77 \\
\hline RMS $0.8^{1}$ & 27.0 & 35.0 & 59.5 & 0.207 & 0.143 & $3.84 \mathrm{e}-8$ & $1.46 \mathrm{e}-8$ & 0.85 \\
\hline RMS $0.8^{2}$ & 27.9 & 31.5 & 70.4 & 0.333 & 0.244 & $4.30 \mathrm{e}-8$ & $1.96 \mathrm{e}-8$ & 1.67 \\
\hline RMS 1.0 & 27.6 & 34.0 & 65.8 & 0.309 & 0.197 & $4.18 \mathrm{e}-8$ & $1.72 \mathrm{e}-8$ & 1.40 \\
\hline
\end{tabular}

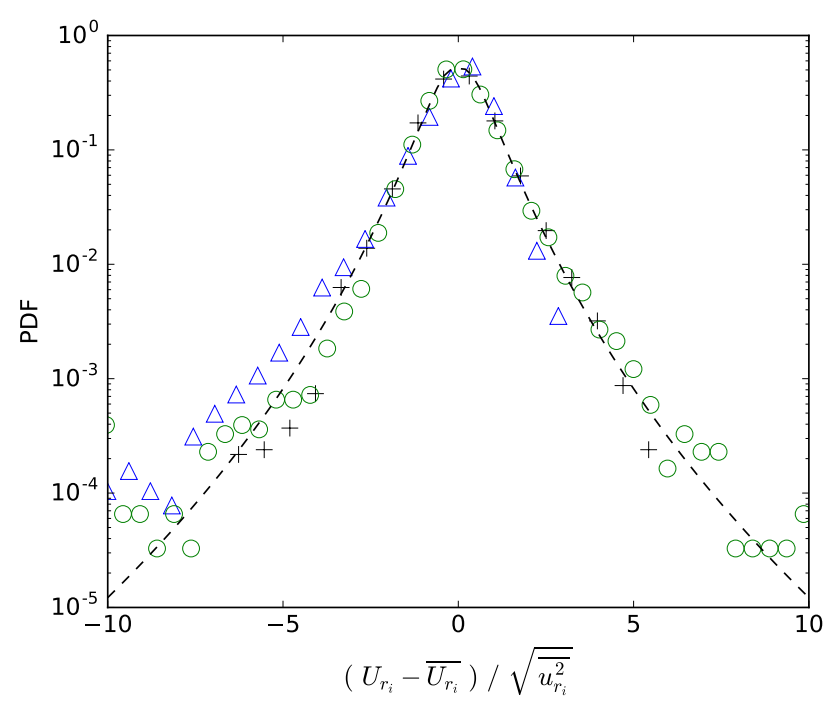

FIG. 6. Probability density functions of the normalized relative velocity components. $+: x$ direction, $\triangle: y$ direction, $\circ: z$ direction, dashed line: equation (1) with $\mathrm{s}=0.62$.

Sherwood number $S h_{F}$ is determined by

$$
S h_{F}=2+\frac{S h_{0}-2}{F_{M}\left(B_{M}\right)}
$$

where $S h_{0}$ is the Sherwood number for a non vaporizing sphere and $F_{M}$ a factor that accounts for the blowing of the vapor film caused by the Stefan flow (Abramzon and Sirignano [22]). The Reynolds numbers of the droplets in our experiment being low, typically $R e_{d}<$ $10, S h_{0}$ is estimated by using the Clift correlation (Clift et al. [36]):

$$
S h_{0}=1+\left(1+R e_{d} S_{c g}\right)^{\frac{1}{3}} f\left(R e_{d}\right) ;
$$

where $f\left(R e_{d}\right)=1$ for $R e_{d} \leq 1, f\left(R e_{d}\right)=R e_{d}^{0.077}$ for $1<R e_{d} \leq 400$ and $S_{c g}$ stands for the Schmidt number of the surrounding gas film. The thermophysical average properties in the gas film around the droplet are calculated at a temperature and a vapor mass fraction of reference, based on the so-called 1/3 law (Hubbard et al. [37], Yuen and Chen [38]) :

$$
T^{r}=\frac{2}{3} T^{s}+\frac{1}{3} T^{\infty} ; \quad Y_{e}^{r}=\frac{2}{3} Y_{e}^{s}
$$

The gas in the film around the droplet is considered as a mixture of the humid air of the room and the vapor of diethyl ether. The Reynolds number $R e_{d}$ used in equation (4), is the one based on the viscosity $\mu_{g}^{r}$ of the gas film around the droplet in the reference conditions, and the density of humid air at infinite $\rho_{h a}^{\infty}$ :

$$
R e_{d}=\frac{\rho_{h a}^{\infty}\left|\mathbf{U}_{\mathbf{d}}-\mathbf{U}\right| d}{\mu_{g}^{r}}
$$

In the absence of turbulence, the surrounding gas velocity $\mathbf{U} \approx \overrightarrow{0}$ and taking the instantaneous values of $\mathbf{U}_{\mathbf{d}}$ measured along the trajectory by holography yields a quasi-linear " $\mathrm{d}^{2}$ " law that reproduces well the experimental " $\mathrm{d}^{2}$ " Lagrangian evolution. As already noted, diethyl ether vaporizes very fast, which cools the humid air around the droplets and causes its condensation at their surface. The water mass condensation rate $\dot{m}_{w}$ was therefore included in the model to account for this effect. With turbulence (Marié et al. [3]), $\mathbf{U}$ being not null, we used for $\mathbf{U}_{\mathbf{d}}-\mathbf{U}$ the instantaneous relative velocity $\mathbf{U}_{\mathbf{r}}$ seen by the droplets along their trajectories and inferred from our holographic measurements. The implementation of this instantaneous velocity in the convective term (equations (3)-(4)) was shown 

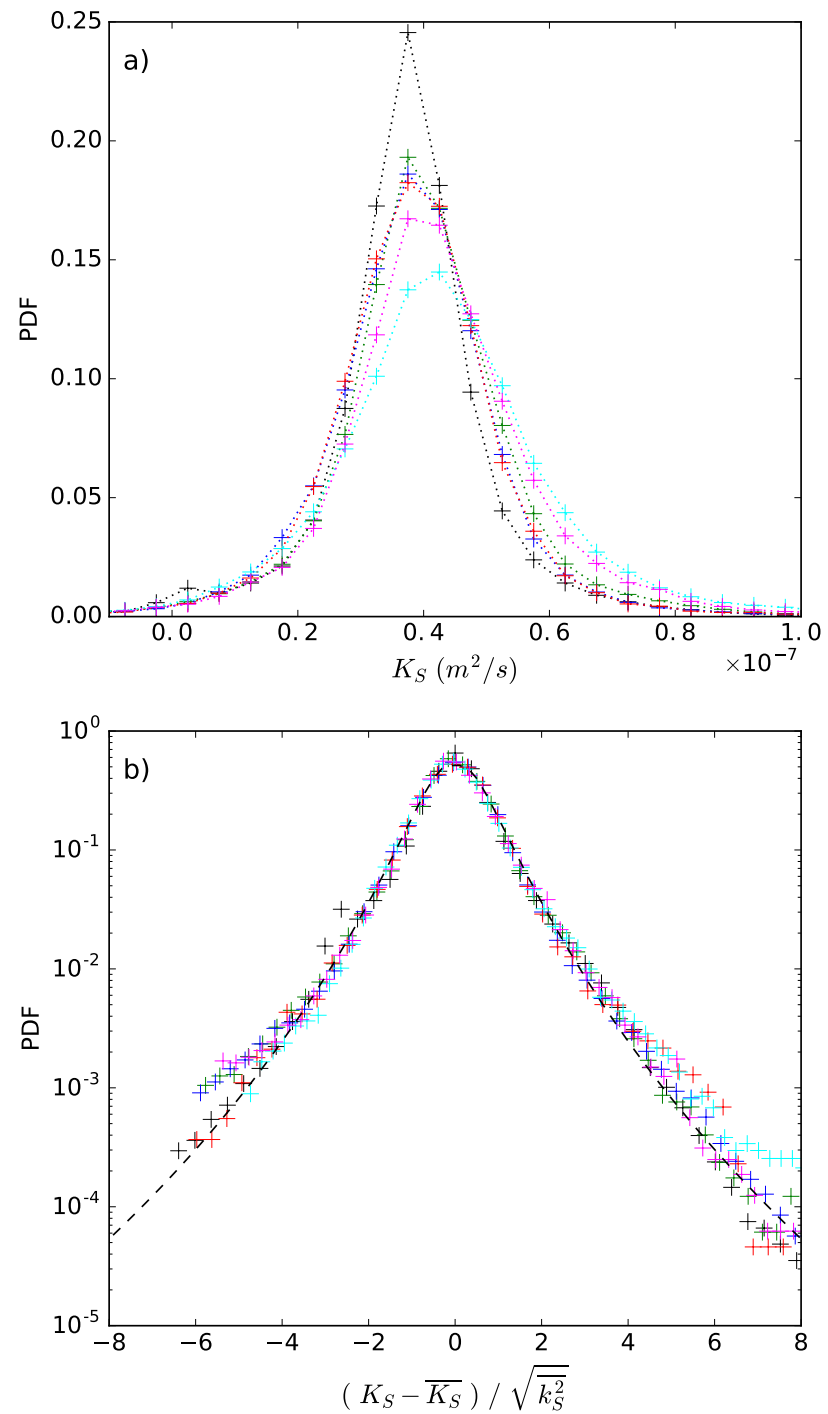

FIG. 7. Probability density function of evaporation rate $K_{S}$ (a) and normalized evaporation rate (b) for the runs RMS 0.2 (+), RMS 0.4 $(+)$, RMS 0.6 (+), RMS 0.8 ${ }^{1}(+)$, RMS $0.8^{2}(+)$, RMS 1.0 (+). The black dashed line is the PDF of Eq (1) with $\mathrm{s}=0.62$.

to reproduce rather well the measured evaporation rates for certain of the ten droplets trajectories reported, but to greatly underestimate these rates for the three of them where droplets were submitted to intense variations of the relative velocity. These results suggest that the turbulence seen by the droplets influence their evaporation and that the convective effects induced by this turbulence are not always well predicted by considering the dependency on the instantaneous relative velocity alone. We will now examine whether these trends are confirmed by the present statistics. The PDFs and statistics on a large number of samples presented in figure $7 \mathrm{a}$ and in table IV clearly show that increasing the turbulence contributes to the increase of the mean evaporation rate and the occurrence of high evaporation rates relative to this mean. Compared with our previous study, droplets diameter and thus Stokes numbers are smaller (section II B). Their Reynolds numbers are also typically lower: $R e_{d} \leq 3$, against 1-20 in the previous flow situation, meaning that convective effects induced

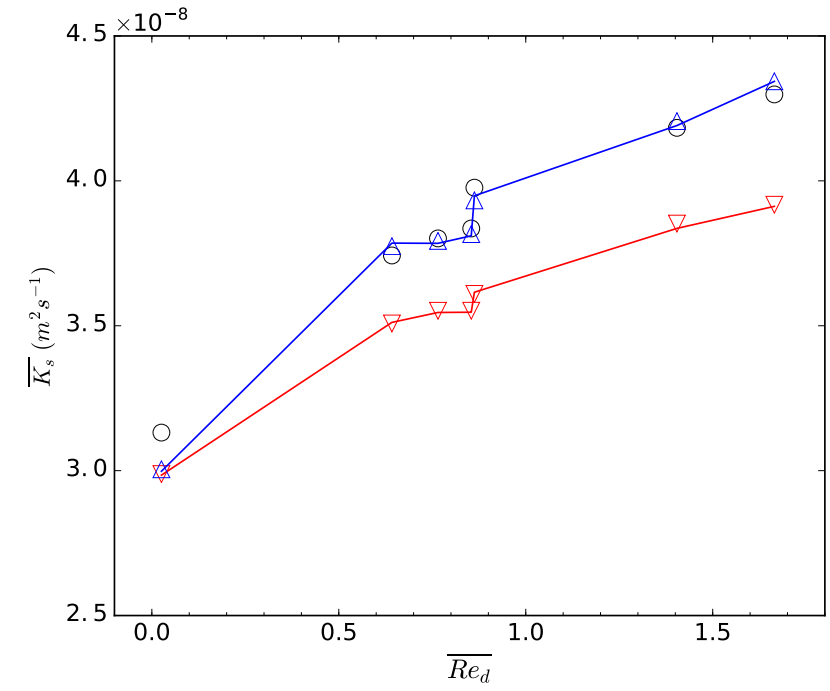

FIG. 8. Mean statistical Lagrangian surface evaporation rate as a function of the mean Reynolds number based on the instantaneous relative velocity. $\circ$ : measurements, red solid line: calculation with $\left|\mathbf{U}_{\mathbf{d}}-\mathbf{U}\right|=U_{r} ; \nabla$ : calculation with $\left|\mathbf{U}_{\mathbf{d}}-\mathbf{U}\right|=\overline{U_{r}} ; \triangle$ : calculation with $\left|\mathbf{U}_{\mathbf{d}}-\mathbf{U}\right|=\overline{U_{r}}+u_{r}^{\prime}$; blue solid line: calculation with equation (9).

by the turbulence are also lower.

Running the model with $T^{\infty}, R_{h}^{\infty}, \overline{d_{I}}$, and the statistical Lagrangian velocities of table IV as input, indicates that whatever the turbulent flow conditions, $S_{c g}$ lies between 1.8 and $1.9, B_{M}$ between $0.41-0.43$ and $F_{M}\left(B_{M}\right)$ is close to 1.07 . It means that the convective Sherwood number $S h_{F}$, as defined by equation (3) and (4), essentially depends on $R e_{d}$. The surface evaporation rates $K_{s}=-\dot{d}^{2}$ can therefore be written in the form

$$
K_{s}=K_{s_{0}} \operatorname{Sh}_{F}\left(R e_{d}\right)
$$

where $K_{s_{0}}=4 \frac{\rho_{g}}{\rho_{e}} D_{v e} \log \left(1+B_{M}\right)$ is almost constant for a given turbulent flow condition. The resulting mean Lagrangian evaporation rate, calculated over all the samples corresponding to one turbulent condition, is expressed as

$$
\overline{K_{s}}=K_{s_{0}} \overline{S h_{F}\left(R e_{d}\right)}
$$

Figure 8 compares the mean statistical experimental evaporation rate $\bar{K}_{S} \exp$ reported in table IV (black circles) with the mean evaporation rate $\overline{K_{S}\left(U_{r}\right)}$ calculated with the norm $U_{r}$ of the instantaneous relative velocity (red solid line). The small experimental increase observed for $\overline{R e_{d}}=0.85$ is principally linked to the 1 degree temperature difference between runs RMS 0.4 and 0.8 and gives an order of magnitude of this effect on our measurements. Note that humidity rate also varies between the two runs, but the effect of these variations is much weaker. We see that, whatever the mean relative Reynolds number $\overline{R e_{d}}$, the model captures well this temperature effect but substantially underestimates the whole experimental data. Also, we note that $\overline{K_{S}\left(U_{r}\right)}$ is very close to the mean evaporation rate $\overline{K s\left(\overline{U_{r}}\right)}$ (red triangles) that is obtained with the mean Lagrangian relative velocity $\left(\left|\mathbf{U}_{\mathbf{d}}-\mathbf{U}\right|=\overline{U_{r}}\right)$. The fact that $\overline{K_{S}} \exp >\overline{K s\left(\overline{U_{r}}\right)}$ and that $\overline{K_{s}\left(U_{r}\right)} \approx \overline{K s\left(\overline{U_{r}}\right)}$ indicate that the relative velocities fluctuations statistically contribute to increase the average convective effect and that 


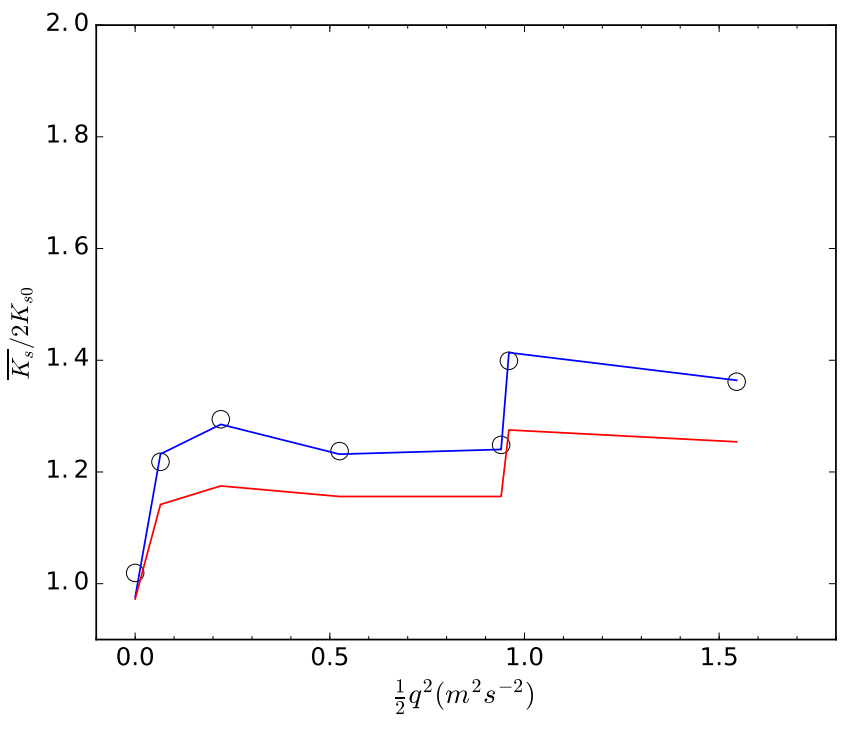

FIG. 9. The mean statistical Lagrangian evaporation rate $\overline{K_{s}}$ normalized by the value without convective effect $2 K_{s 0}$ as a function of the box turbulent kinetic energy. ○: measurements; blue line: $\overline{K_{S}}$ calculated with equation (9); red line: $\overline{K s\left(\overline{U_{r}}\right)} / 2 K_{s}^{0}$.

this increase is not taken into account with $\left|\mathbf{U}_{\mathbf{d}}-\mathbf{U}\right|=U_{r}$ in equation (6). The parameter that quantifies the strength of these relative velocities fluctuations is the RMS turbulent velocity $u_{r}^{\prime}$. So we can expect that this turbulent velocity, added to the mean relative velocity, generates an additional turbulent convective effect that increases the average evaporation rate. To see whether this could explain the difference between $\overline{K_{S}} \exp$ and $\overline{K_{S}\left(U_{r}\right)}$, we have calculated the mean evaporation rate that would result from the mean relative velocity plus the relative fluctuations strength: $\left|\mathbf{U}_{\mathbf{d}}-\mathbf{U}\right|=\overline{U_{r}}+u_{r}^{\prime}$. The results thus obtained (blue triangles) exhibit excellent agreement with the experimental data, confirming the above statement. We deduce that to agree with the Lagrangian statistics, the effective Reynolds number to be considered in the convective term for the simulation is

$$
\operatorname{Re}_{d}^{t u r b}=\operatorname{Re}_{d}\left(U_{r}\right)\left(1+\frac{u_{r}^{\prime}}{\overline{U_{r}}}\right)
$$

where the second term in parenthesis quantifies the importance of $u_{r}^{\prime}$ relative to $\overline{U_{r}}$. This leads to an evaporation rate in figure 8 (blue solid curve) that on average well reproduces the experimental data.

These findings statistically validate what was suspected in Marié et al. [3], namely that the convective term is not accurately predicted by the film model, even when the norm of the instantaneous relative velocity is considered. The reason is that the modelling is based on quasi-steady assumptions and mean values of heat/mass coefficients within boundary layers Clift et al. [36] that can hardly capture the disturbances generated by the turbulence. This turbulence induces changes in norm and direction of relative velocity which may be rapid as illustrated in Marié et al. [3], forcing the thermal and mass boundary layers to continuously reorient with the flow. This likely affects the transfers inside the boundary layers (Kleis and RiveraSolorio [39], Sirignano [40]) and contributes to the evaporation enhancement. In this respect the formulation of equation (9), although empirical, turns out to be the simplest approach to include the statistical turbulence effects in the film model. This approach is shown to work well over the range of parameter investigated here: Schmidt number $\approx 2$ and droplets Reynolds number $\leq 3$. This range of parameter is obviously too restricted to conclude whether equation (9) can be used for more general flow conditions, at high Reynolds numbers in particular. Investigating these high Reynolds number conditions would require to inject larger droplets. But larger droplets trajectories are closer to ballistic and there residence time in the box is often too short to observe significant effect and to obtain reliable Lagrangian statistics. Also the fluids tested must vaporize very quickly which restrains the Schmidt number range allowed in this study. Despite these limitations, our results show that the turbulence in the box increases the mean evaporation rate via the $\overline{U_{r}}$ and $u_{r}^{\prime}$ it generates at the droplets scale. However, the increase of the evaporation rate with the box turbulence is not monotonic. The reason is that $\overline{U_{r}}$ and $u_{r}^{\prime}$ don't monotonically increase with the RMS turbulent velocity produced by the woofers (see tables II, IV). This is attributed to the fact that injection conditions, like the initial droplet diameter, the velocity and the turbulence level at the injector exit, may differ from run to run. This is for example the case of the two runs RMS $0.8^{1}$ and $0.8^{2}$ where diameters are $59.5 \mu \mathrm{m}$ and $70.5 \mu \mathrm{m}$, respectively. These differences might influence the way the droplets respond to turbulence and explain the trends that are observed. To quantify the effect of the box turbulence on evaporation, the mean evaporation rate $\overline{K_{S}}$ normalized by its value without convective effect $2 K_{s}^{0}$ has been plotted versus the turbulent kinetic energy $0.5 q^{2}$ in figure 9 . We see that this normalized mean evaporation rate which is close to 1 for the free falling situation, increases with the turbulent kinetic energy up to 1.4 for the runs RMS $0.8^{2}$ and RMS 1.0, representing an increase of $40 \%$. The part of this percentage due to $\overline{U_{r}}$ and represented by $\overline{K s\left(\overline{U_{r}}\right)} / 2 K_{s}^{0}$ in the figure is about $30 \%$. Although substantial, these increases are small in comparison with the experiments with droplets suspended in box of turbulence $[4,25,41]$. This comes from the nature of the fluids investigated in these experiments (usual fuels) and of their physical properties which are different, with an impact on the Schmidt and Damkhöler numbers. But more importantly, droplet diameters are generally greater $(1.5-2 \mathrm{~mm})$, resulting in turbulent Reynolds number of the order of 50-100 and much higher convective effects. Moreover being immobilized, droplets are submitted to stronger relative turbulent velocities at the same equal turbulent kinetic energy.

\section{SUMMARY OF THE MAIN FINDINGS}

These experiments have been performed with the objective to statistically confirm our previous findings [3], and to check whether ether droplets released in a quasi isotropic homogeneous turbulence with zero mean flow, on average, vaporize faster than in free falling conditions. To this end, Lagrangian statistics were computed on a large amount of trajectories representing about 1.3 million samples and covering various RMS turbulent velocities between 0.2 and 1.0 $\mathrm{ms}^{-1}$. The instantaneous droplets coordinates and diameter constituting each trajectory were measured from sequences of digital holograms processed with an Inverse problem approach (IPA). The time required to process the 554 sequences recorded for our statistics, and estimated to 57 years on a single $\mathrm{CPU}$, has been drastically reduced to allow this study. This was made possible by implementing the IPA code in Python language for a High Performance Computing system and running the code on various nodes of 16 or 32 cores within a few weeks.

The statistics on the droplets dynamics show that for high turbulence velocity fluctuations $\left(0.8 \mathrm{~ms}^{-1}\right)$ and high $R e_{\lambda}$, the PDFs of the 3 normalized droplets velocity components superpose rather well onto a Gaussian distribution, with however a small asymmetry and a sligthly higher mean velocity in the vertical direction. The reason is 
that droplets released at the top of the turbulence box enter the box when turbulence is preferentially oriented downward. The PDfs of their 3 normalized acceleration components also superposes rather well. Their shapes resembles the ones found for inertial particles by Qureshi et al. [35] and are in close agreement with the statistical distribution proposed by these authors, taking the same global parameter shape $s=0.62$. The instantaneous relative velocity seen by the droplets was inferred, like in our previous works [1, 3], from the droplet trajectories measured by holography, via the equation of motion. This relative velocity is on average vertical as expected with our injection conditions, with RMS fluctuations in the 3 directions close to each other, reflecting a certain isotropy of the fluctuating relative motion. The PDFs of the 3 normalized relative velocity components exhibit a shape similar to the one observed for the droplets acceleration components.

Regarding the evaporation rate, the statistics indicate that the mean Lagrangian evaporation rate increases with the mean and fluctuating relative velocity seen by the droplets. The latter two quantities are found to increase with the box RMS turbulent velocity. However this increase is not strictly monotonic which is attributed to variations in injection conditions. Results were confronted to the predictions of the simple evaporation film model proposed and tested in Marié et al. [2]. The use of the instantaneous norm of the relative velocity to estimate the convective effects in the Sherwood number yields predictions that significantly underestimate the measured mean Lagrangian evaporation rate. This validates what was assumed in Marié et al. [3] and shows that the model cannot capture the kinematic, thermal, and mass transfers disturbances induced by the turbulence at the droplet surface when using only this norm. In contrast, an excellent agreement with the data is obtained when the Sherwood number is calculated from a Reynolds number based on the norm of the mean relative velocity plus its RMS fluctuation. Adding this RMS fluctuation is thus a simple empirical formulation to correct the film model and to account for the evaporation enhancement caused by the turbulence. This formulation works well within the parameter range explored here: Schmidt number $\approx 2$, Reynolds number $\leq 3$. Further investigations would be necessary to test its possible validity for other flows situations, at higher Reynolds numbers in particular. The evaporation rates measured for the strongest turbulence levels, (turbulent velocities between 0.8 and $1 \mathrm{~ms}^{-1}$ ) prove to be $40 \%$ higher than the one in free falling conditions. We conclude that the effect of turbulence on the droplet evaporation via the convective effects it generates at the droplet scale is substantial.

\section{ACKNOWLEDGMENTS}

This work was granted access to the HPC ressources of the PMCS2I-FLMSN, "Fédération Lyonnaise de Modélisation et Sciences Numériques". The authors express their gratitude to Anne Cadiou and Laurent Pouilloux for their technical assistance.
[1] D. Chareyron, J.-L. Marié, C. Fournier, J. Gire, N. Grosjean, L. Denis, M. Lance, and L. Méès, Testing an in-line digital holography "inverse method" for the Lagrangian tracking of evaporating droplets in homogeneous nearly-isotropic turbulence, New J. Phys. 14 (2012), 043039.

[2] J.-L. Marié, N. Grosjean, L. Méès, M. Seifi, C. Fournier, B. Barbier, and M. Lance, Lagrangian measurements of the fast evaporation of falling diethyl ether droplets using in-line digital holography and a high speed camera, Exp. Fluids 55 (2014), doi:10.1007/s00348-014-1708-6.

[3] J.-L. Marié, T. Tronchin, N. Grosjean, L. Méès, O. Öztürk, C. Fournier, B. Barbier, and M. Lance, Digital holographic measurement of the lagrangian evaporation rate of droplets dispersing in a homogeneous isotropic turbulence, Exp. Fluids 58 (2017), doi: 10.1007/s00348-016-2292-8.

[4] M. Birouk and I. Gökalp, Current status of droplet evaporation in turbulent flows, Prog. Energ. Combust. Sci. 32, 408 (2006).

[5] D. B. Spalding, Combustion of fuel particles, Fuel 30, 121 (1951).

[6] S. Pope, Turbulent Flows (Cambridge Univ. Press, 2000).

[7] S. Balachandar and J. Eaton, Turbulent Dispersed Multiphase Flow, Annu. Rev. Fluid Mech. 42, 111 (2010).

[8] A. Berlemont, M.-S. Grancher, and G. Gouesbet, On the lagrangian simulation of turbulence influence on droplet evaporation, Int. J. Heat Mass Transf. 34, 2805 (1991).

[9] J. Reveillon and F.-X. Demoulin, Effects of the preferential segregation of droplets on evaporation and turbulent mixing, J. Fluid Mech. 583, 273 (2007).

[10] S. Sahu, Y. Hardalupas, and A. Taylor, Interaction of droplet dispersion and evaporation in a polydispersed spray, J. Fluid Mech. 846, 37 (2018).

[11] C. Goepfert, J.-L. Marié, D. Chareyron, and M. Lance, Characterization of a system generating a homogeneous isotropic tur- bulence field by free synthetic jets, Exp. Fluids 48, 809 (2010).

[12] F. Toschi and E. Bodenschatz, Lagrangian properties of particles in turbulence, Annu. Rev. Fluid Mech. 41, 375 (2009).

[13] B. Gopalan, E. Malkiel, and J. Katz, Experimental investigation of turbulent diffusion of slightly buoyant droplets in locally isotropic turbulence, Phys. Fluids 20 (2008), 095102.

[14] J. Lu, J.-P. Fugal, H. Nordsiek, E.-W. Saw, R.-A. Shaw, and W. Yang, Lagrangian particle tracking in three dimensions via single-camera in-line digital holography, New J. Phys. 10 (2008), 125013.

[15] J. Katz and J. Sheng, Applications of holography in fluid mechanics and particle dynamics, Annu. Rev. Fluid Mech. 42, 531 (2010).

[16] D. Nguyen, D. Honnery, and J. Soria, Measuring evaporation of micro-fuel droplets using magnified DIH and DPIV, Exp. Fluids 50, 949 (2011).

[17] F. Soulez, L. Denis, C. Fournier, E. Thiébaut, and C. Goepfert, Inverse-problem approach for particle digital holography: accurate location based on local optimization, J. Opt. Soc. Am. A 24, 1164 (2007).

[18] F. Soulez, L. Denis, E. Thiébaut, C. Fournier, and C. Goepfert, Inverse problem approach in particle digital holography: outof-field particle detection made possible, J. Opt. Soc. Am. A 24, 3708 (1951)

[19] J. Gire, L. Denis, C. Fournier, E. Thiébaut, F. Soulez, and C. Ducottet, Digital holography of particles: benefits of the "inverse-problem" approach, M. Sci. Technol. 19 (2008), 074005.

[20] C. Fournier, L. Denis, E. Thiébaut, T. Fournel, and M. Seifi, Inverse problems approaches for digital hologram reconstruction, in Three Dimensional Imaging, Visualization, and Display 2011, Vol. 8043 (Orlando, United States, 2011) pp. 1-14. 
[21] R.-B. Bird, W.-E. Stewart, and E.-N. Lightfoot, Transport Phenomena (John Wiley, 1960).

[22] B. Abramzon and W.-A. Sirignano, Droplet vaporization model for spray combustion calculations, Int. J. Heat Mass Transf. 32, 1605 (1989).

[23] W. Hwang and J.-K. Eaton, Creating homogeneous and isotropic turbulence without a mean flow, Exp. Fluids 36, 444 (2004).

[24] H. Lian, G. Charalampous, and Y. Hardalupas, Preferential concentration of poly-dispersed droplets in stationary isotropic turbulence, Exp. Fluids 54, 1525 (2013), doi 10.1007/s00348-0131525-3.

[25] U. Patel and S. Sahu, Effect of air turbulence and fuel composition on bi-component droplet evaporation, International Journal of Heat and Mass Transfer 141, 757 (2019).

[26] S. Lin and R. Reitz, Drop and spray formation from a liquid jet, Annu. Rev. Fluid Mech. 30, 85 (1998).

[27] E. R. Lee, Microdrop generation (CRC Press, 2003).

[28] M. Seifi, C. Fournier, N. Grosjean, L. Méès, J.-L. Marié, and L. Denis, Accurate 3D tracking and size measurement of evaporating droplets using in-line digital holography and "inverse problems" reconstruction approach, Opt. Express 21 (2013), DOI:10.1364/OE.21.027964.

[29] L. Méès, N. Grosjean, D. Chareyron, J.-L. Marié, M. Seifi, and C. Fournier, Evaporating droplet hologram simulation for digital in-line holography setup with divergent beam, J. Opt. Soc. Am. A 30, 2021 (2013).

[30] M. Born and E. Wolf, Principles of Optics (Pergamon press, 1980).

[31] M. Seifi, C. Fournier, L. Denis, D. Chareyron, and J.-L. Marié, $3 \mathrm{~d}$ reconstruction of particle holograms: a fast and accurate multi-scale approach, J. Opt Soc. Am A 29 (2012), DOI:
10.1364/JOSAA.29.001808.

[32] N. Mordant, A.-M. Crawford, and E. Bodenschatz, Threedimensional structure of the Lagrangian acceleration in turbulent flows, Phy.Rev.Lett. 93, 214501 (2004).

[33] G.-A. Voth, A. Laporta, A.-M. Crawford, J. Alexander, and E. Bodenschatz, Measurement of particle acceleration in fully developed turbulence, J. Fluid. Mech. 469, 121 (2002).

[34] N. M. Qureshi, M. Bourgoin, C. Baudet, A. Cartellier, and Y. Gagne, Turbulent transport of material particles: An experimental study of finite size effects, Phys. Rev. Lett. 99, 184502 (2007).

[35] N. M. Qureshi, U. Arrieta, C. Baudet, A. Cartellier, Y. Gagne, and M. Bourgoin, Acceleration statistics of inertial particles in turbulent flow, The European Physical Journal B 66, 531 (2008).

[36] R. Clift, J.-R. Grace, and M.-E. Weber, Bubbles, Drops and Particles (Academic Press, 1978).

[37] G.-L. Hubbard, V.-E. Denny, and A.-F. Mills, Droplet evaporation: Effects of transients and variable properties, Int. J. Heat Mass Transf. 18, 1003 (1975).

[38] M. Yuen and L.-W. Chen, On drag of evaporating droplets, Combut. Sci. and Technol. 14, 147 (1976).

[39] S. J. Kleis and I. Rivera-Solorio, Time Scales for Unsteady Mass Transfer From a Sphere at Low-Finite Reynolds Numbers , Journal of Heat Transfer 125, 716 (2003).

[40] W.-A. Sirignano, Fluid Dynamics and Transport of Droplets and Sprays (Cambridge University Press, 2010).

[41] C. Verwey and M. Birouk, Experimental investigation of the effect of droplet size on the vaporization process in ambient turbulence, Combustion and Flame 182, 288 (2017). 